\title{
Evaluación en Línea: Herramientas, Limitaciones y Alternativas en un Contexto de
} Pandemia

\author{
Online assessment: Tools, Limitations and Alternatives in a Pandemic Context
}

Jon Xabier Basogain-Urrutia ${ }^{1}$

\section{¿ EDICIÓN:

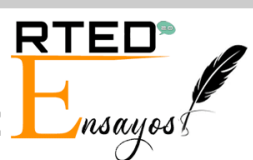

Recibido: 21/junio/2021

Aceptado: 21/agosto/2021

Publicado: 29/agosto/2021

\section{País}

${ }^{1}$ España

\section{IIIII Institución}

${ }^{1}$ Toki Eder Ikastola

\section{@Correo Eletrónico \\ 1jonxabier_basogain001@ehu.eus}

\section{(D) ORCID}

${ }^{1}$ https://orcid.org/0000-0002-1454-6798

\section{Citar así: $\mathcal{C l}_{\text {APA } / \text { IEEE }}$}

Basogain-Urrutia, J. (2021). Evaluación en Línea: Herramientas, Limitaciones y Alternativas en un Contexto de Pandemia.. Revista Tecnológica-Educativa Docentes 2.0, 10(2), 30-41 https://doi.org/10.37843/rted.v10i2.243

J. Basogain-Urrutia, "Evaluación en Línea: Herramientas, Limitaciones y Alternativas en un Contexto de Pandemia.", RTED, vol. 10, n. $^{\circ}$, pp. 30-41, ago. 2021.

\section{Resumen}

La pandemia originada por el SARS-CoV-2 ha afectado a muchos aspectos del desarrollo del ser humano, destacando entre ellos la educación del alumnado de todas las edades, teniendo que incorporar abruptamente elementos de la docencia virtual en entornos donde ni siquiera se contemplaban. La evaluación durante la pandemia ha sido mayoritariamente desarrollada en línea, evidenciando así los escasos conocimientos de la comunidad docente respecto a los métodos de evaluación en línea. Ha surgido en el mundo de la educación la necesidad de conocer y utilizar las herramientas de la evaluación en línea. En respuesta a esta situación, se ha recogido en este ensayo una revisión de las herramientas de evaluación y de supervisión de exámenes en línea disponibles en la comunidad educativa, así como un análisis de sus limitaciones y alternativas a las mismas. Además, se ha propuesto un modelo, adaptado a la edad y a las características del aprendiz, de los sistemas de evaluación en línea como marco referencial dirigida a la comunidad educativa responsable de la formación de las futuras promociones en estos tiempos pospandémicos.

Palabras clave: Evaluación en línea, herramientas, limitaciones, alternativas.

\section{Abstract}

The pandemic caused by SARS-CoV-2 has affected many aspects of human development, and especially the education of students of all ages, having to abruptly incorporate elements of virtual teaching in environments where they were not even considered. Assessment during the pandemic has been mostly developed online, evidencing the limited knowledge of the teaching community regarding online assessment methods. The need to know and use online assessment tools has arisen in the world of education. In response to this situation, a review of the online assessment and test proctoring tools available in the educational community, as well as an analysis of their limitations and alternatives to them, has been collected in this essay. In addition, a model, adapted to the age and characteristics of the learner, of online assessment systems has been proposed as a frame of reference addressed to the educational community responsible for the training of future promotions in these postpandemic times.

Keywords: Online assessment, tools, limitations, alternatives. 


\section{Introducción}

Existen multitud de recursos en línea creados con el objetivo de aprender, enseñar y evaluar en línea. Se podría pensar que su auge se debe al creciente uso de internet en los últimos años o incluso a la situación sanitaria actual creada por la COVID-19. Aunque, en cierto modo, sí se ha dado tal incremento en un corto periodo de tiempo, el origen del aprendizaje en línea a nivel universitario tiene su origen mucho más atrás. En 1999, por ejemplo, la Universidad de La Rioja ya proponía impartir titulaciones en línea (Moreno \& Santiago, 2003).

Hoy en día, la oferta es inmensamente más amplia si se compara con la de hace hace 20 años, pues ya nos encontramos con universidades nacidas como instituciones en línea. Un claro ejemplo es el de la UNIR (Universidad Internacional de La Rioja) también llamada "La Universidad en Internet", que ofrece estudios íntegramente online y aglutina un gran número de alumnos, cerca de 45.000 , pese a que propone evaluaciones finales presenciales (Universidad Internacional de La Rioja, 2015; Periódico La Rioja, 2019). Otro ejemplo similar es el de la Universidad Internacional de Valencia, que propone en sus másteres evaluaciones totalmente en línea; aunque debido a la pandemia, esta decisión también se ha extendido a los estudios de grado (Universidad Internacional de Valencia, 2020, 2021a; 2021b).

Otras universidades, que no nacieron como universidades en internet, como la Universidad Católica de Ávila, han ido incorporando a su oferta modalidades no presenciales (ya sea blearning o e-learning) y con ello, elementos de la docencia virtual (Universidad Católica de Ávila, 2021). La Universidad del País Vasco (UPV/EHU), si bien no ofrece estudios no presenciales, cuenta desde hace más de 10 años, con Moodle como apoyo a la docencia presencial, un entorno de aulas virtuales usado por muchas instituciones a nivel mundial (Ros, 2008). Fuera del contexto universitario, junto a Moodle, una de las herramientas más empleadas es Google Classroom; incluída dentro del paquete que Google pone a disposición de las escuelas, y ha sido objeto de numerosos estudios (e.g., Kraus et. al., 2019).
Estos datos evidencian que, incluso mucho antes de la pandemia originada por el SARSCoV-2, la mayoría de las instituciones ya contaban con plataformas virtuales mediante las cuales se creó una nueva realidad donde la docencia presencial, salvo rara excepción, siempre cuenta con apoyos en la nube (Fernández-Pampillón, 2009). Así pues, se podría pensar que, cuando se requirió, transitar a una modalidad totalmente virtual pudo ser sencillo. La realidad, sin embargo, resultó ser diferente. Hubo que integrar, de emergencia, elementos de la enseñanza a distancia en modalidades donde no estaban contemplados, lo cual conllevó un laborioso proceso y un gran esfuerzo de las instituciones (Abreu, 2020).

El proceso resultó especialmente complejo para aquellas edades donde el aprendizaje a distancia es prácticamente inconcebible, como la educación infantil y primaria. Esta circunstancia se vio agravada por la llamada brecha digital, pues, al requerirse ciertos recursos mínimos para la enseñanza en línea (e.g., ordenador, cámara, micrófono y buena conexión a internet) no siempre se pudo garantizar la disposición de los mismos para todo el alumnado (Hoz et al., 2021). Uno de los grandes retos fue la formación del profesorado, muchas veces cuestionada, en un contexto donde se transitó tan abruptamente a la modalidad en línea, sin contar con tiempo suficiente para adquirir recursos y habilidades suficientes (Romero et. al., 2017; CóndorHerrera, 2020). Este reto vino acompañado por la dificultad de realizar evaluaciones en línea.

Impartir clases resultó relativamente sencillo, gracias a herramientas de videoconferencias integradas en entornos elearning, como BlackBoard Collaborate Ultra, u otras, que, no habiendo sido creadas con fines académicos demostraron ser adecuadas, como Zoom (Hill, 2019, Nadezhda, 2020; Cheung, 2021). Fue más complejo supervisar los exámenes en línea, a causa de la falta de experiencia de la comunidad educativa en esta área. Se desconocían las herramientas de monitoración de exámenes en línea, así como las experiencias de implementación institucional y manejo a nivel de usuario; esta situación de incertidumbre reabrió el debate sobre la validez de los exámenes finales (Jiménez et al., 2017; Kharbat, 2021; López-Alvarez, 2013). 
En las siguientes líneas, se describen las principales plataformas de e-learning (Moodle, BlackBoard Learn y Google Classroom), así como las técnicas y herramientas para asistir en la supervisión de exámenes en línea (conocido como e-proctoring en inglés) y las alternativas a dichos recursos o métodos. Finalizando con la sección de conclusiones donde se presenta un modelo o marco referencial de los métodos de exámen más adecuados para cada etapa educativa.

\section{Desarrollo}

A continuación, se presentan las características de las plataformas e-learning más empleadas por instituciones educativas para después ver qué opciones de evaluación en línea ofrecen. También se exponen las técnicas de supervisión de exámenes en línea o e-proctoring, así como las alternativas a los mismas.

Plataformas de e-learning y sus características. Las instituciones educativas de todo el mundo emplean diferentes aplicaciones para gestionar sus entornos de e-learning, pero tres de estas herramientas son especialmente conocidas debido a su amplio uso: Moodle, BlackBoard Learn y Google Classroom. Resulta importante conocerlas si se quiere ahondar en los recursos ofrecidos por estas plataformas para afrontar el reto del seguimiento de la formación y evaluación en línea.

1.1. Moodle. Se trata de una herramienta libre, totalmente gratuita. Su nombre viene del acrónimo en inglés: Modular Object-Oriented Dynamic Learning Environment (Ximenez, 2020). Es un aula virtual donde, además de poder colgar contenidos y archivos, se pueden incluir otro tipo de recursos que ofrece esta aplicación. Se pueden añadir herramientas de todo tipo para evaluación en línea, como tareas, foros, tareas revisadas por pares, libros, periódicos $\mathrm{y}$ encuestas, entre otros muchos recursos (Pruneda, 2020). Una de las principales ventajas de Moodle es que, al integrarse recursos elaborados por terceros, se pueden crear un sinfín de entornos y experiencias. En la figura 1 se muestra el aspecto de un curso de Moodle.

\section{Figura 1}

Aspecto de un curso de Moodle, una vez se ha accedido al mismo

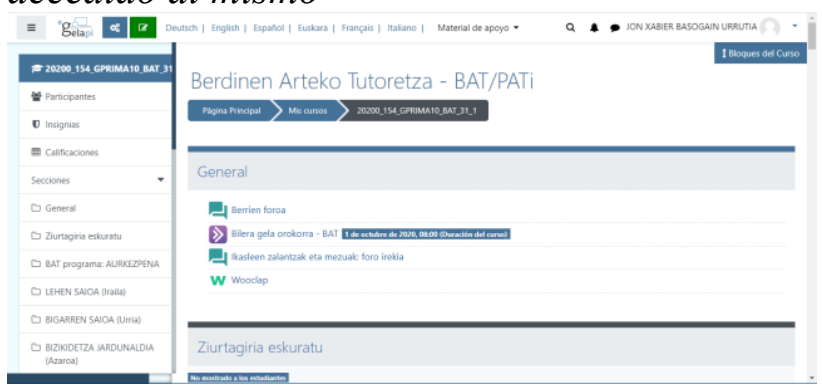

Nota. En la imagen se observa un curso, aun en oculto para estudiantes. Entre los recursos dispuestos, se ven tres tipos de actividades: (a) foros, (b) instancia de BlackBoard Collaborate para reuniones, (c) instancia de Wooclap. Elaboración propia en el servicio Moodle eGELA-PI de la UPV/EHU (2021).

La mayoría de las instituciones educativas, mediante sus plataformas de e-learning ofrecen una amplia batería de recursos, varios de ellos, destinados a la evaluación en línea y a apoyar la docencia presencial. Muchos otros, sin embargo, tienen como objeto asistir en el diseño instruccional, creando materiales de aprendizaje interactivos como H5P o paquetes SCORM. En la figura 2 se puede observar el conjunto de recursos recogidos en Moodle.

\section{Figura 2}

Recursos disponibles en Moodle

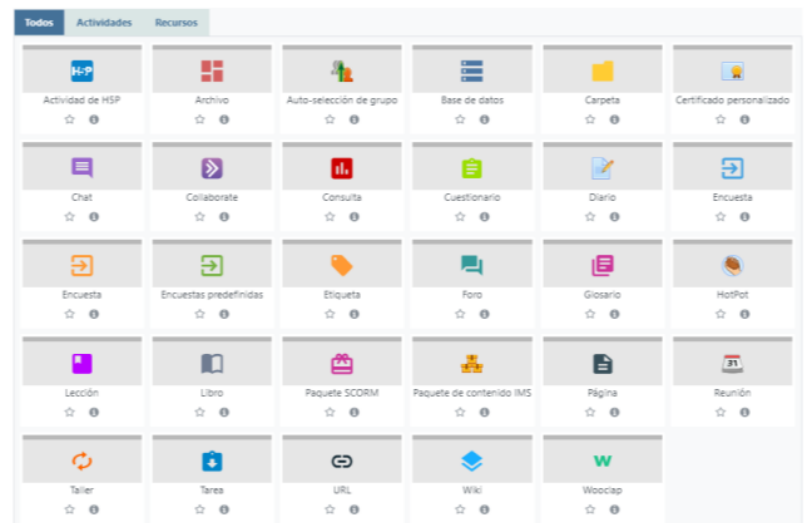

Nota. Los recursos disponibles en Moodle más comunes son los presentados en la imagen, la mayoría están incluidos en todas las plataformas Moodle. Elaboración propia en el servicio de Moodle eGELA-PI de la UPV/EHU (2021).

Entre los recursos disponibles en Moodle, y más concretamente entre aquellos que son calificables o están orientados de algún modo u otro a la evaluación, existen cinco que destacan sobre los demás por su amplio uso y su facilidad 
de uso. Estos no necesariamente pasan por la entrega de un texto o respuesta de un examen, sino que pueden estar basados en aspectos más abiertos e informales como puede ser el foro. Los siguientes recursos son los más empleados (Moodle, 2016):

- Tarea: es una instancia donde los alumnos pueden subir una tarea previamente descrita, además, se puede restringir el tipo de archivos aceptados, añadir una fecha máxima de entrega, e incluso extender individualmente a cada alumno el plazo de entrega. Como alternativa a subir un archivo, dicho recurso puede ser configurado en la modalidad de "texto en línea", donde el alumno escribe dentro de Moodle empleando su propio editor de texto.

- Taller: es un recurso similar a "tarea" donde los alumnos pueden realizar la entrega de una tarea que será revisada y evaluada no solo por el docente, sino también por uno de los compañeros (también se conoce como P2P, tarea por pares o peer to peer en inglés).

- Cuestionario: generalmente se emplea como el sustituto de un examen tradicional, donde se pueden incluir preguntas de diverso tipo (test, respuesta corta, respuesta larga, unir términos, arrastrar, rellenar huecos...). Asimismo, se puede seleccionar un tiempo límite para terminar el cuestionario, así como aleatorizar el orden de las preguntas, o incluso que el examen o cuestionario se genere automáticamente en base a una bolsa de preguntas.

En Moodle, además, existe la opción de calificar recursos que, a priori, pueden no parecer destinados a la evaluación en línea. Algunos de dichos recursos son los foros (donde se puede proponer al alumnado que debata sobre un tema argumentando sus participaciones para, después, evaluar ese foro), y los libros o lecciones donde se pueden incluir preguntas después de explicaciones teóricas (Moodle, 2016). La figura 3 muestra cómo quedan estos cinco recursos más empleados para la evaluación en un curso Moodle.

\section{Figura 3}

Integración de los recursos evaluables más usados en Moodle

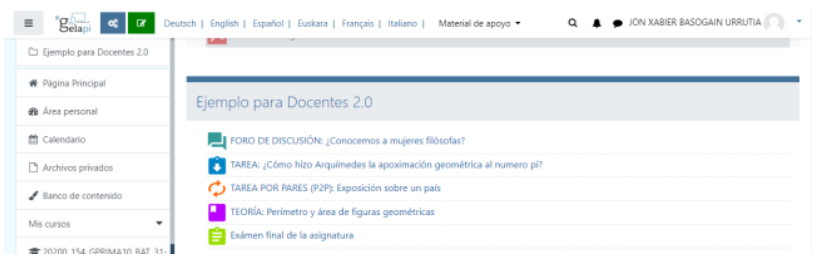

Nota. Muestra cómo se presentan los recursos calificables expuestos en Moodle, con el modo de edición desactivado. Elaboración propia en el servicio Moodle eGELA-PI de la UPV/EHU (2021).

Como se puede observar, esta herramienta dota tanto al docente como al alumno de recursos muy útiles orientados a una evaluación en línea, ya sea empleando pruebas de evaluación continua, como un portafolio, o con pruebas como el examen final. Además, el cálculo automático (previa configuración) de las calificaciones finales sin necesidad de usar herramientas externas como Microsoft Excel, facilita el trabajo de los agentes implicados en la evaluación, donde la configuración del libro de calificaciones permite seguir al momento el progreso del estudiante (Díaz-Quintero, 2020).

\subsection{Blackboard Learn. BlackBoard}

Learn es una herramienta LMS (Learning Management System) muy similar a Moodle, pero de pago (Gladilina et al., 2020). Incluye en su oferta de servicios la herramienta de videoconferencias BlackBoard Collaborate Ultra, compatible con otras plataformas LMS. En el caso de Moodle, por ejemplo, se mostraba (figura 2) entre los recursos disponibles el icono de BlackBoard Collaborate; en Moodle es posible habilitar instancias de esta herramienta de videoconferencias mediante el plugin creado para ello, pero única y exclusivamente si se dispone de una licencia de BlackBoard Collaborate (Moodle, 2021).

Esta plataforma de e-learning, BlackBoard Learn, ofrece prácticamente las mismas características de Moodle (foros, actividades/tareas, cuestionarios, entre otros) a diferencia de dos aspectos de uso y navegación, distintivos de este entorno, relacionados con cómo se visualizan los contenidos de un curso y con la categorización de las aulas virtuales en "cursos" y "organizaciones": 
- Navegación: en estos cursos se navega empleando el menú lateral, el cual incluye vínculos a una determinada página del curso, mientras en Moodle se puede organizar un curso por temas, pestañas o semanas, pero no existe tal sistema de páginas independientes. Es cierto que versiones más recientes de Moodle incluyen un menú vertical a modo de índice, pero de ningún modo funciona como un sistema de páginas independientes como BlackBoard Learn.

- Distinción entre cursos y organizaciones: las aulas virtuales se pueden clasificar en cursos y organizaciones. Esta característica particular no afecta al aspecto del aula virtual ni al tipo de recursos que se pueden incluir, sino a la gestión y navegación por el LMS, puesto que los cursos e instituciones se muestran separados en la página de inicio. Hay espacios destinados asignaturas (un aula virtual al uso) y espacios denominados organizaciones, los cuales son cursos, pero destinados a un determinado grupo de usuarios y funcionan como un aula general para dicho grupo de personas (e.g., todos los alumnos de primer curso o todos los alumnos de una titulación). No tiene un objetivo académico y persigue la comunicación con estas personas, así como facilitar aspectos de gestión (BlackBoard, 2021a).

\subsection{Google Classroom. Esta herramienta} dista más de las anteriores. Así como Moodle y BlackBoard Learn ofrecen un amplio abanico de recursos, esta ofrece únicamente seis actividades posibles para incluir en el curso. Esta posible carencia, sin embargo, se ve suplida al poder interactuar desde este entorno de aulas virtuales con el entorno de Google y poder añadir ficheros directamente desde, por ejemplo, Google Drive. Estos son los seis recursos ofrecidos (Tabla 1).

\section{Tabla 1}

\begin{tabular}{ll} 
Recursos de Google Classroom \\
TAREA & $\begin{array}{l}\text { Se concibe como en Moodle y BlackBoard Learn; es una instancia donde los alumnos añaden un archivo (texto, } \\
\text { audio, vídeo...) en respuesta al trabajo solicitado por el docente. Es calificable, ofreciendo la posibilidad enviar } \\
\text { comentarios de retroalimentación y crear rúbricas. A diferencia de las otras herramientas e-learning descritas, } \\
\text { no permite entregar un texto en línea, siempre ha de usarse un editor externo no alojado en Classroom. }\end{array}$ \\
\hline CuESTIONARIO & $\begin{array}{l}\text { Se concibe como una tarea en donde se ha de responder a un cuestionario (Google Forms). Debido a que permite } \\
\text { convertir el formulario en un examen y establecer puntuaciones a las preguntas, puede emplearse como tal. }\end{array}$
\end{tabular}

\begin{tabular}{ll} 
PREgUNTA & $\begin{array}{l}\text { Es una instancia calificable en donde los alumnos deben responder a una pregunta publicada por el profesor. } \\
\text { Funciona de modo similar a una tarea debido a su configuración que únicamente permite al profesor ver las } \\
\text { respuestas de los alumnos. }\end{array}$ \\
\hline MAterial & $\begin{array}{l}\text { Se emplea con la finalidad de subir archivos al aula virtual. Estos pueden ser de cualquier tipo, al ser Google } \\
\text { Classroom compatible con todas las extensiones de archivo más comunes. }\end{array}$ \\
\hline PUBLICACIÓN & $\begin{array}{l}\text { Permite importar recursos (tareas, preguntas...) de otros cursos del mismo docente. Este tipo de importación de } \\
\text { recursos instantáneo no está disponible en otros entornos LMS donde la recuperación de instancias de otros } \\
\text { cursos resulta laboriosa en comparación. }\end{array}$ \\
\hline TEMA & $\begin{array}{l}\text { Su función es crear diferentes apartados en el aula virtual o curso, permitiendo anidar determinados materiales } \\
\text { y contenidos del curso, así como tareas, dentro de cada uno de los apartados creados. }\end{array}$
\end{tabular}

Nota. Dichos recursos, ofrecen posibilidades al estar vinculados a todo el ecosistema Google. Elaboración propia empleando los iconos originales de Google Classroom, (2021).

A pesar de tener únicamente seis recursos para configurar el aula, las oportunidades de crear cursos con muchos materiales se ven exponencialmente aumentadas al trabajar en el ecosistema de Google, pudiendo así configurar formularios, presentaciones, hojas de cálculo u otros recursos como tareas y recursos del aula (Zhang, 2016). Es decir, además de poder subir archivos o incluir recursos en la red como vídeos 
o enlaces, es posible basar las tareas en las aplicaciones ofrecida por Google.

A diferencia de las otras plataformas de elearning ofrece dos vistas del curso. La figura 4 muestra la vista denominada "Trabajo de clase" donde se pueden ver las secciones del curso y los recursos disponibles en cada uno. La otra vista, accesible en una pestaña denominada "Tablón" (ver figura 5), presenta una especie de bandeja de entrada con las últimas novedades del curso; cada vez que se publica un trabajo, un material o un profesor manda un mensaje, aparece ahí; también ofrece personalizar el aspecto de cada curso, funcionalidad restringida en Moodle $y$ BlackBoard Learn, donde el aspecto se personaliza para toda la institución (GómezGoitia, 2020).

\section{Figura 4}

Aspecto de la vista "Trabajo de clase" en Google Classroom

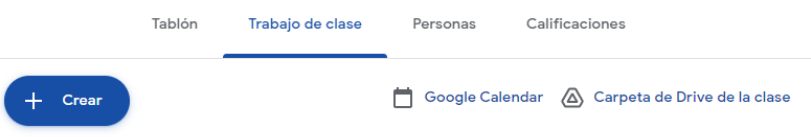

Present continuous

(1) Where am !?

Vocabulary

目 Interactive Test

目 Green is not always the right answer

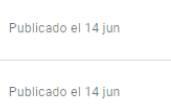

Nota. Esta vista muestra una disposición de los elementos más convencional y similar a la que ofrece Moodle. Elaboración propia, empleando los servicios de Google Classroom (2021).

\section{Figura 5}

Aspecto de la vista "tablón" de Google Classroom

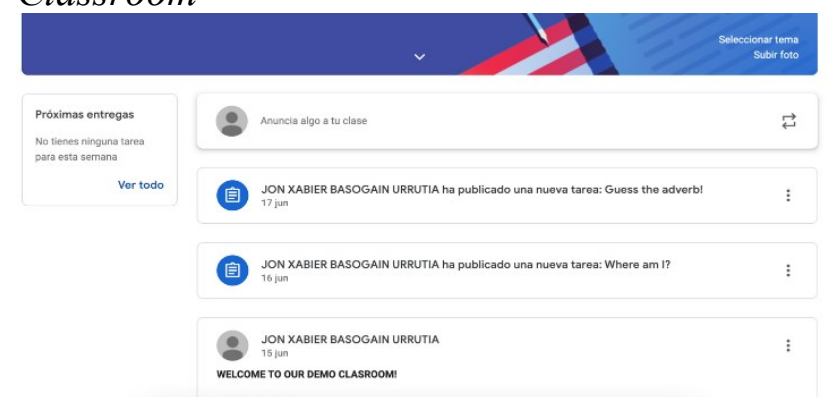

Nota. Se notifica al alumnado mediante correo electrónico cada vez que se publica algo en el tablón. Elaboración propia empleando los servicios de Google Classroom, (2021).
En general, todas las plataformas LMS, independientemente de su estructura, ofrecen un conjunto mínimo de recursos para llevar a cabo la evaluación en línea. En el aspecto económico, sin embargo, únicamente la plataforma BlackBoard Learn requiere una licencia de uso. En el caso de Google Classroom, su uso es gratuito incluso para instituciones, mientras empleen el servicio de Google for Education; si se usa con una cuenta personal de Gmail, ha de marcarse una casilla indicando que no se está usando con fines académicos dentro de una institución (Iftakhar, 2016).

Exámenes en línea: recursos para evitar el fraude en pruebas finales en vivo y sus limitaciones. Como ya se ha indicado, se puede efectuar una enseñanza no presencial de una manera adecuada empleando entornos e-learning mediante el uso de pruebas de evaluación continua (como foros, tareas...). El problema surge cuando se desean emplear recursos con objeto de simular o sustituir un examen de evaluación final, garantizando tanto la honestidad académica como la ausencia de fraude académico durante el transcurso de la prueba (e.g. la persona realizando el examen es el propio estudiante).

En pruebas de portafolio, es evidente que se pueden emplear técnicas antifraude como los programas de detección de plagio como SafeAssign de Blackboard (Blackboard, 2021b). Pero el problema surge cuando la prueba es en vivo (a modo de examen), donde se quiere garantizar que el estudiante no copia en el examen, no tiene apuntes a mano, y es él mismo quien realiza el examen. Es precisamente aquí donde aquellas universidades no concebidas para impartir docencia online tuvieron mayores problemas a causa de que no contaban con infraestructuras suficientes para desarrollar este tipo de pruebas con total garantía.

Existen, hoy día, diversas herramientas, recursos y programas, algunos incluso basados en inteligencia artificial, que permiten supervisar los exámenes en línea con mayor facilidad. Cada uno tiene características diferentes; mientras algunos se basan en restringir funcionalidades del ordenador, otros monitorizan el ordenador y registran todo lo que sucede en el mismo (si se ha 
copiado y pegado texto, si se ha abierto otra pestaña...) así como identifican al alumno y buscan posibles conductas fraudulentas mediante un sistema biométrico. A continuación, se describen las herramientas y técnicas más empleadas:

\subsection{Conexión en directo mediante} videoconferencia. Algunas universidades, así como centros no universitarios optaron por llevar a cabo videoconferencias con el alumno mientras este realizaba el examen en línea. En este caso, se empleaba una herramienta de videoconferencias donde el alumno debía mantener tanto el micrófono como la cámara encendida mientras se desarrollaba la prueba, asegurando una supervisión en vivo. (Corell et. al., 2020).

Algunas instituciones, además, pedían a los alumnos mostrar su entorno, así como su DNI (Documento Nacional de Identidad) antes de comenzar el examen, todo ello en aras de garantizar la ausencia de otras personas y verificar su identidad. Este tipo de modalidades de supervisión también son oportunas si se quiere facilitar una vía de comunicación en vivo entre los agentes implicados en la evaluación por si hubiera problemas de cualquier índole durante el desarrollo de la prueba.

\subsection{Programas de bloqueo del} escritorio. Estas herramientas, como Respondus Lockdown Browser (Respondus), consisten en bloquear el escritorio. Esta aplicación requiere estar instalada en el ordenador del estudiante, para, una vez iniciada, ocultar todos los botones del sistema (barra de inicio del ordenador, fecha...). Respondus funciona a modo de navegador, en el cual solo se puede abrir una pestaña, generalmente el Campus Virtual de la institución examinadora. Mientras el programa está abierto, no se puede abrir ni ver ninguna otra aplicación del ordenador, ni copiar o pegar texto. Tampoco se permitirá acceder al examen desde un navegador diferente a Respondus (Questroom School of Bussiness, 2016).

\subsection{Programas de monitorización y} supervisión en vivo (e-proctoring). Los programas de supervisión en línea, como Smowl, basado en una inteligencia artificial, monitorizan en vivo toda la actividad desarrollada en el ordenador y capturan tanto imagen como sonido del entorno donde se desarrolla la prueba. El alumno, el primer día dentro del campus virtual, debe tomarse tres fotos para posteriormente ser analizadas por un sistema basado en inteligencia artificial. Este sistema reconocerá su cara en los próximos exámenes, asegurándose así de que siempre es el estudiante quien realiza las pruebas.

Durante el examen Smowl realiza dos actividades simultáneas: toma fotos del alumno cada cierto tiempo, ejecutando el reconocimiento facial, así como comprobando la ausencia de segundas personas o voces; asimismo, también garantiza la presencia del alumno frente a la pantalla (detecta la ausencia de personas). Por otro lado, se monitoriza en vivo la actividad del ordenador del examinado (qué programas tiene abiertos, así como si hay pestañas abiertas en el navegador...). Si se han abierto otros programas, toma capturas de pantalla, y reconoce, además, en caso de que se haya copiado texto, desde qué web o programa se ha copiado, detallando exactamente qué texto ha sido copiado.

Finalmente, se genera un informe con todos los registros del examen, permitiendo al docente decidir si con la evidencia presentada por Smowl, ha habido fraude o no. Este tipo de programas tienen ajustes personalizables dotando así a la institución de libertad para establecer aquello que se entenderá como fraude. Por ejemplo, se puede decidir grabar audio o no, si se debe registrar si hay más de una pantalla, teclado o ratones conectados, o si se debe avisar cuando el estudiante lleve auriculares, entre otras muchas funciones (Labayen et al., 2014).

\subsection{Combinación de métodos}

anteriores. Algunas instituciones consideran insuficiente emplear un solo método de los anteriormente descritos, optando por combinarlos. Por ejemplo, una combinación habitual es supervisar el examen simultáneamente mediante programa de bloqueo de escritorio, y una conexión en vivo mediante videoconferencia con el estudiante (Universidad Católica de Ávila, 2020a).

2.5. Limitaciones. Dichos métodos pueden parecer útiles para garantizar una evaluación en línea sin fraude, por lo que puede resultar difícil escoger cuál funciona mejor. Si bien todas son útiles a su manera, lo más importante será adecuarse tanto al nivel académico en el cual se lleva a cabo la evaluación como al tipo de alumnado. A modo de resumen, a continuación, se muestra resumidamente un 
cuadro comparativo (tabla 2) de ventajas, así como de limitaciones de las diferentes herramientas de monitorización de exámenes en línea.

\section{Tabla 2}

Comparativa de ventajas y limitaciones de las diferentes herramientas de monitorización de exámenes en vivo

$\begin{array}{lllll}\begin{array}{l}\text { CONEXIÓN MEDIANTE } \\ \text { VIDEOCONFERENCIA }\end{array} & \text { BLOQUEO DE ESCRITORIO } & \begin{array}{l}\text { MONITORIZACIÓN } \\ \text { SUPERVISIÓN EN } \\ \text { (E-PROCTORING) }\end{array} & \begin{array}{l}\text { COMBINACIÓN } \\ \text { VIVO }\end{array} & \text { DE MÉTODOS }\end{array}$

Se puede ver qué es lo que
hace el alumno,
garantizando la identidad
del estudiante, así como
evitando que haya otras
personas en la sala. Existe
conexión en directo para
consultar dudas.

(A)

Se garantiza el bloqueo de actividades fraudulentas como copiar y pegar texto o tener otros programas abiertos; a la par que se bloquea también la opción de navegar en internet fuera del campus virtual.

\begin{tabular}{|c|c|c|}
\hline $\begin{array}{l}\text { esulta } \\
\text { onitorizar lo que el } \\
\text { suario hace en su } \\
\text { ddenador } \\
\text { formación en internet o } \\
\text { apuntes...), así como } \\
\text { mpoco se puede } \\
\text { arantizar que no se } \\
\text { bpia/pega texto. En caso } \\
\text { haber muchos } \\
\text { tudiantes, resulta } \\
\text { borioso supervisar } \\
\text { luchas sesiones de } \\
\text { deoconferencia }\end{array}$ & $\begin{array}{l}\text { identidad del estudiante, } \\
\text { ni tampoco se puede } \\
\text { garantizar que no se esté } \\
\text { consultando información } \\
\text { en soportes no digitales } \\
\text { (apuntes en papel, por } \\
\text { ejemplo) o en otros } \\
\text { dispositivos distintos al } \\
\text { usado para examinarse, } \\
\text { los cuales no cuentan con } \\
\text { estos programas } \\
\text { instalados. }\end{array}$ & $\begin{array}{l}\text { ser supervisados } \mathrm{p} \\
\text { humano que ha de } \mathrm{c} \\
\text { si ha habido fraude } \\
\text { puesto que } \\
\text { programas genera } \\
\text { informe basado } \\
\text { fotografías to } \\
\text { aleatoriamente dura } \\
\text { examen y el re } \\
\text { obtenido de } \\
\text { monitorización } \\
\text { escritorio (aplica }\end{array}$ \\
\hline
\end{tabular}

Queda garantizada la identidad del estudiante gracias al control biométrico y se analizan posibles conductas fraudulentas, a la par que se cuenta con un informe de monitorización de escritorio.

Contar con más de una vía ofrece cierto margen en caso de que una falle. La combinación más empleada, debido a su capacidad de supervisión consiste en combinar una herramienta de bloqueo de escritorio, junto a la videoconferencia.

Ciertos programas de bloqueo de escritorio, debido a que solo permiten acceder al campus virtual de la institución, requieren que la sesión de videoconferencia sea abierta mediante un navegador convencional, permitiendo únicamente la comunicación por audio entre alumno y supervisor. Esto puede dificultar el desarrollo de la prueba, así como la labor de supervisión.

simultáneamente.

Durante el transcurso de los exámenes en vivo realizados mediante internet, pueden ocurrir diversos problemas como interrupción de la conexión a internet o del servicio eléctrico, fallos de hardware (cámara o micrófono, principalmente) así como desconexiones no intencionadas. Dichos fallos perjudican gravemente el progreso de las pruebas; podrían solventarse empleando alternativas al examen final (desarrolladas en la siguiente sección) las cuales no requieran conectarse un día y hora concretos durante un tiempo prolongado, sino que permitan al estudiante desarrollar sus habilidades sin depender en tan alto grado de factores externos.

Nota. Este cuadro compara los cuatro tipos de monitorización en este ensayo, todos ellos tienen tanto limitaciones como ventajas, elaboración propia en base a los datos expuestos anteriormente (Labayen et al., 2014; Questroom School of Bussiness, 2016; Corell et al., 2020; Universidad Católica de Ávila, 2020a).

\section{Alternativas a exámenes o pruebas finales escritas}

Se ha visto que el mayor problema para evitar el fraude en la evaluación en línea yace en los exámenes escritos finales, en los cuales los alumnos han de responder correctamente a una serie de preguntas teórico-prácticas, empleando los conocimientos adquiridos durante el transcurso de la actividad lectiva. Para responder a esta problemática, se proponen tres alternativas a las pruebas o exámenes finales.

3.1. Porfolio de actividades. Se trata de poner en valor el trabajo realizado en la asignatura durante el curso, buscando desarrollar pruebas que pongan a prueba los conocimientos de los estudiantes, haciéndoles buscar soluciones y adquirir competencias disciplinares e interdisciplinares (Gregori \& Martín-Rojo, 2011). Este tipo de actividades pueden ser exposiciones, resoluciones de casos, e investigaciones.

\subsection{Actividades realizadas en clase. La} evaluación en línea no debe por qué atender únicamente a producciones escritas de actividades o exámenes. Es igualmente útil, a la par que enriquecedor, crear tanto debates como discusiones en clase donde el alumno deba 
exponer sus argumentos a favor o en contra de una pregunta o problemática propuesta por el docente. Así, el alumno deberá posicionarse, argumentar y opinar basándose en hechos $\mathrm{y}$ conocimientos teóricos. Dichas actividades pueden ser tanto formales (previamente planificadas) como informales (surgidas en clase).

3.3. Proyecto final. Si se desea llevar a cabo un trabajo o un proyecto final, a modo de culminación del curso, siendo este independiente de las actividades del portafolio, una buena alternativa puede ser el proyecto final. Este proyecto puede ser un trabajo de investigación (e.g. exponer los descubrimientos más relevantes de Arquímedes) o una actividad más creativa como puede ser mandar cartas a los personajes de un libro leído en clase desde el punto de vista de un amigo del personaje, comentando algo sucedido en la historia. También puede tratarse de un proyecto donde haya un producto final (como contar un cuento empleando Scratch, por ejemplo) o una iniciativa STEAM (Science, Technology, Engineering, Art and Mathematics). Este tipo de iniciativas se aproxima a lo conocido como el Aprendizaje Basado en Proyectos (ABP) donde los alumnos realizan un recorrido de aprendizaje, culminado con la presentación de un proyecto (Trujillo, 2015; Jiménez et al., 2017).

\section{Conclusión}

Se presentan en este apartado qué métodos son más adecuados para cada etapa educativa, atendiendo a las características del desarrollo del alumnado, así como a la competencia digital, entre otros. Se atenderán las luces y sombras del proceso de adaptación a una modalidad no presencial con el objetivo de perfilar un enfoque óptimo, adecuado a las necesidades y características de cada contexto educativo.

Como se ha descrito previamente, el proceso de transición a la docencia y evaluación en línea fue dispar en el mundo, incluso dentro de cada país. Había instituciones mejor preparadas, en particular aquellas creadas con el fin de enseñar en línea, ya que contaban con herramientas de monitorización de pruebas online (e-proctoring). Otras instituciones, sin embargo, tuvieron mayores problemas, pues contaban con entornos e-learning, pero no pudieron garantizar la correcta evaluación en línea, al no contar con sistemas de supervisión exámenes. En consecuencia, se vieron forzadas a utilizar sistemas de monitorización únicamente mediante videoconferencia, teniendo este método grandes limitaciones.

Además, si bien se ha presentado esta problemática desde un punto de vista más universitario, la pandemia conllevó el traslado a una modalidad no presencial de todos los niveles educativos. Esto supuso que tanto profesores como alumnos no familiarizados con entornos elearning se viesen forzados a adoptar sistemas de enseñanza y evaluación en línea en entornos donde nunca antes se había utilizado. Por ejemplo, en los primeros cursos de Educación Primaria supuso todo un reto enseñar a leer y a escribir a distancia, lo cual ya está siendo objeto de numerosas investigaciones (e.g., Aristega, 2021).

Cuando se habla de evaluación en línea, se toma en consideración el contexto en el cual se realiza esa evaluación, atendiendo a los siguientes aspectos: (a) edad de los estudiantes, (b) etapa educativa, (c) medios de e-learning y eproctoring disponibles. En base a dichos aspectos,

resulta evidente la imposibilidad de evaluar con los mismos métodos $\mathrm{y}$ herramientas de evaluación a alumnos de infantil, digitalmente no competentes, o a alumnos de secundaria, con amplia experiencia en el uso de recursos digitales.

Con el objetivo de escoger el mejor método de evaluación en línea, adecuándose a la edad de los aprendices y a su contexto, se propone el siguiente modelo basado en la competencia digital del alumnado, sus características evolutivas y las propiedades de las herramientas de monitorización de exámenes en línea (Piaget, 1975; Labayen et al., 2014; Questroom School of Bussiness, 2016; Cabezas et. al. 2017; Corell et al., 2020; Universidad Católica de Ávila, 2020). Dicho modelo se estructura como una progresión que comienza con métodos más sencillos en edades tempranas hasta alcanzar un nivel de complejidad superior en edades universitarias.

En el caso de educación infantil y los primeros cursos de educación primaria, la interacción con el aprendiz (aun si esta es por videoconferencia), el portafolio de actividades y 
las prácticas realizadas en clase permiten la evaluación en línea, ofreciendo recursos y evidencias del aprendizaje suficientes. Son edades (3 a 8 años) donde los alumnos, en la mayoría de las ocasiones, aún tienen grandes dificultades a la hora de conectarse por sí mismos a las clases, responder a un cuestionario en línea o desarrollar cualquier actividad propia del aprendizaje en línea. La dependencia del adulto es alta para este rango edad, en consecuencia, hay que diseñar técnicas de evaluación que se ajusten al nivel de autonomía del aprendiz.

Ya en los últimos cursos de educación primaria (9-12 años), incluso en el primer curso de educación secundaria (12-13 años), puede resultar útil y adecuado llevar a cabo pruebas de evaluación final en una plataforma de e-learning en la cual el alumno se mueva fácilmente por haberla empleado durante el curso; pero este tipo de pruebas no deben cobrar especial valor sobre la nota final. En primaria el portafolio de actividades sería una buena alternativa para garantizar la adquisición de competencias. Si se proponen pruebas finales, estas pueden ser monitoreadas en línea o no, pero en tal caso, conviene no emplear métodos demasiado invasivos para el alumno, puesto que aún es joven $\mathrm{y}$ debe ir tomando confianza con los métodos virtuales conforme adquiere competencias en los mismos. Un buen sistema de monitorización podría ser el de seguir el examen mediante una videoconferencia en vivo donde pueda preguntar a su profesor viendo así que no está solo.

En educación secundaria, sobre todo a partir de los primeros cursos y bachillerato (1418 años) podría ir haciéndose una transición hacia métodos de e-proctoring más complejos respecto a la videoconferencia. Los cuestionarios o exámenes finales pueden ser adecuados, pero sin obviar del todo el portafolio de actividades. Por ejemplo, los programas de bloqueo de escritorio pueden resultar una buena opción, incluso, en los cursos superiores, si se combinan con el sistema de vigilancia mediante videoconferencia.

En contextos universitarios (alumnos mayores de 18 años) para estudios de grado, posgrado, títulos propios y cualquier otra actividad docente universitaria es tanto posible como recomendable optar por sistemas de eproctoring basados en inteligencia artificial para las pruebas finales. Estos sistemas garantizan la identidad del estudiante, además de monitorizar aquello que hace el estudiante en su entorno físico (movimientos del estudiante, ausencia del mismo, uso de gafas o auriculares) y en su ordenador (abrir otras pestañas, pegar texto desde otra página o editor de texto). Gracias a estos sistemas se cuenta con un gran volumen de información para avalar la seguridad de estas pruebas. En cualquier caso, sin embargo, resulta vital acompañar estas pruebas con proyectos, portafolios y el resto de las alternativas propuestas.

Las edades sugeridas anteriormente son orientativas y siempre han de tenerse en cuenta las características del grupo de estudiantes examinado. Aunque podría parecer adecuado hacer coincidir estos cambios en el método de supervisión de exámenes en línea con cambios de etapa académica (transición de Educación Primaria a Educación Secundaria, por ejemplo), lo ideal es que no sucedan exactamente al mismo tiempo, para facilitar así el ajuste al nuevo contexto académico y adecuarse mejor a las características evolutivas y madurativas del aprendiz. Esta regla se rompe con el comienzo de los estudios universitarios, generalmente a los 18 años de edad, donde ya se considera adecuado hacer coincidir cambios en el método de supervisión en línea con los cambios de etapa educativa. Se ha elaborado una tabla resumen del método propuesto (Tabla 3 ).

Tabla 3

Resumen del modelo de evaluación en línea.

\begin{tabular}{|c|c|c|}
\hline EDAD & $\begin{array}{c}\text { MÉTODO DE } \\
\text { SUPERVISIÓN DE LA } \\
\text { PRUEBA FINAL }\end{array}$ & $\begin{array}{l}\text { INSTRUMENTOS DE } \\
\text { EVALUACIÓN EN LÍNEA }\end{array}$ \\
\hline $3-7$ & $\begin{array}{l}\text { No hay examen o } \\
\text { prueba final. }\end{array}$ & $\begin{array}{l}\text { Énfasis en la interacción } \\
\text { con el alumno } \\
\text { (presencial o por } \\
\text { videoconferencia). } \\
\text { Actividades y proyectos } \\
\text { realizados en clase. }\end{array}$ \\
\hline $8-13$ & Videoconferencia. & Actividades de \\
\hline 14-18 & $\begin{array}{l}\text { Sistemas de bloqueo } \\
\text { de escritorio y/o } \\
\text { videoconferencia. }\end{array}$ & $\begin{array}{l}\text { proyectos, tareas, } \\
\text { actividades en el aula } \\
\text { virtual... }\end{array}$ \\
\hline$>18$ & $\begin{array}{l}\text { Sistemas basados } \\
\text { en inteligencia } \\
\text { artificial. }\end{array}$ & $\begin{array}{l}\text { Porfolio personal. } \\
\begin{array}{l}\text { Pruebas finales o } \\
\text { exámenes. }\end{array}\end{array}$ \\
\hline
\end{tabular}

Nota. Este modelo propone recursos de evaluación y seguimiento de la formación en línea global y a largo plazo, elaboración propia (2021). 
Este modelo propuesto, además de atender las características citadas, debe conocer el marco legal en el cual se desea evaluar el aprendizaje, que garantice los criterios establecidos por las instituciones. En ese sentido, en el contexto universitario, por ejemplo, han de conocerse las directrices y recomendaciones de autoridades políticas e instituciones como ANECA (Grande et. al. 2020). En las pruebas finales con métodos de supervisión en línea, donde alumno debe tener el micrófono y la cámara encendidos, deben conocerse las limitaciones legales con las que cuente dicha supervisión; de hecho, varias universidades como la Universidad Católica de Ávila (UCAV) solicitan a su alumnado firmar un documento de cesión de derechos de imagen para poder examinarse en línea (Universidad Católica de Ávila, 2020b).

No existe un método de evaluación considerado idóneo que ofrezca total garantía, del proceso de evaluación línea. Todos los métodos vistos en este ensayo tienen ventajas $y$ limitaciones, siendo lo verdaderamente importante ser consciente de las mismas, pues solamente así se podrán adoptar las medidas que más se adecúen a cada contexto e institución. Puesto que es cada centro educativo y cada docente quien mejor conoce qué quiere, cuál es su objetivo, cómo desea intervenir y a quién debe dirigir el proceso de evaluación en línea, nunca podrá prevalecer un método sobre otro. Por ello, la labor del ámbito científico es ofrecer datos y recursos a la comunidad escolar y a las instituciones educativas en particular, permitiendo a estas elaborar su propio sistema de evaluación en línea, optimizándolo en base a sus circunstancias, debilidades, fortalezas, oportunidades, y limitaciones.

\section{Referencias}

Abreu, J. L. (2020). Tiempos de Coronavirus: La Educación en Línea como Respuesta a la Crisis. Revista Daena (International Journal of Good Conscience), 15(1), 1-15. http://www.spentamexico.org/v15-n1/A1.15(1)1-15.pdf

Aristega, A., Aristega, J., Angulo, R. \& Masacón, M. (2020). Enseñar y aprender en tiempos de Covid-19. Pro Sciences: Revista de Producción, Ciencias e Investigación, 4(34), 79-86.

Blackboard (2021a). Organizaciones. Ayuda de BlackBoard. https://help.blackboard.com/eses/Learn/Instructor/Ultra/Interact/Organizations
Blackboard (2021b). Submit with SafeAssign. Ayuda de BlackBoard.

https://help.blackboard.com/SafeAssign/Student/Submit SafeAssign

Cabezas González, M., Casillas Martín, S., Sanches-Ferreira, M., \& Teixeira Diogo, F. L. (2017). ¿Condicionan el género y la edad el nivel de competencia digital? Un estudio con estudiantes universitarios. Fonseca, Journal of Communication, 15(15), 109-125 https://doi.org/10.14201/fjc201715109125

Cheung, A. (2021). Language Teaching during a Pandemic: A Case Study of Zoom Use by a Secondary ESL Teacher in Hong Kong. RELC Journal. https://doi.org/10.1177/0033688220981784

Cóndor-Herrera, O. (2020). Educar en tiempos de COVID19. CienciAmérica, 9(2), 31-37. http://201.159.222.118/openjournal/index.php/uti/article/ view/281/419

Corell, A., Grande de Prado, M., García-Peñalvo, F. J., \& AbellaGarcía, V. (2020). Guía de recomendaciones para la evaluación online en las Universidades Públicas de Castilla y León. Version 1.1. https://repositorio.grial.eu/bitstream/grial/2013/1/202005 $01 \% 20$ -

$\% 20$ Recomendaciones\%20valuacio $\%$ CC $\% 81 \mathrm{n} \% 20$ onli ne $\% 20$ para $\% 201$ as $\% 20$ Universidades $\% 20 \mathrm{Pu} \% \mathrm{CC} \% 81 \mathrm{bl}$ icas $\% 20 \mathrm{de} \% 20$ Castilla $\% 20 \mathrm{y} \% 20 \mathrm{Leo} \% \mathrm{CC} \% 81 \mathrm{n} \% 20 \mathrm{~V} 1$. 1.pdf

Díaz-Quintero, M. J. (2020). Configuración de libreta de calificaciones en un curso en la Plataforma Moodle (Versión

https://ridda2.utp.ac.pa/handle/123456789/10305

Fernández-Pampillón Cesteros, A. M. (2009). Las plataformas elearning para la enseñanza y el aprendizaje universitario en Internet. En M. Covadonga \& M. Matesanz del Barrio (Eds). Las plataformas de aprendizaje. Del mito a la realidad (pp. 45-73). Biblioteca Nueva. https://eprints.ucm.es/id/eprint/10682/

Gladilina, I., Pankova, L., Sergeeva, S., Bulochnikova, N., \& Baldin, S. (2020). Learning management system: Integration models of conventional and distance education of students. Eurasian Journal of Biosciences, 14(2), 6153-6159. http://www.ejobios.org/article/learning-managementsystem-integration-models-of-conventional-anddistance-education-of-students-8344

Goitia, J. M. G. (2020). Buena práctica docente para el diseño de aula virtual en Google Classroom. Revista Andina de Educación, 3(1), 64-66. https://doi.org/10.32719/26312816.2020.3.1.7

Grande de Prado, M., García Peñalvo, F. J., Corell, A., \& Abella García, V. (2020). La virtualización causada por el Covid19: recomendaciones para la evaluación. En M. Cid, N. Rajadell, G. Dos Santos (Eds.) Ensinar, avaliar y aprender no ensino superior: Perspetivas internacionais, 231-250. Centro de Investigação em Educação e Psicologia da Universidade de Évora. http://hdl.handle.net/10366/144440

Gregori, E. B., \& De Martín Rojo, E. (2011). Porfolio electrónico: aprender a evaluar el aprendizaje. Editorial UOC. 
Hill, L. (2019). Blackboard Collaborate Ultra: An Online, Interactive Teaching Tool. Academy of Management Learning \& Education, 18(4), 640-642. https://doi.org/10.5465/amle.2019.0027

Hoz, G., Wallace, Y., \& Heredia, M. E. (2021). Alfabetizar a distancia en el inicio de la escuela primaria. Revista Iberoamericana De Tecnología En Educación y Educación En Tecnología, (28), 282-293. https://doi.org/10.24215/18509959.28.e35

Iftakhar, S. (2016). Google classroom: what works and how. Journal of Education and Social Sciences, 3(1), 1218.

Jiménez, C. A., Calderón Badilla, \& Herrero Villarreal, D. (2017). Estrategias adicionales de evaluación en una universidad a distancia: ¿alternativas o complementarias al examen escrito? Cuadernos de Investigación UNED, 9(1), 157 164.

https://www.redalyc.org/jatsRepo/5156/515653587021/5 15653587021.pdf

Kharbat, F. F., \& Daabes, A. S. A. (2021). E-proctored exams during the COVID-19 pandemic: A close understanding. Education and Information Technologies. https://doi.org/10.1007/s10639-021-10458-7

Kraus, G., Formichella, M. M., \& Alderete, M. V. (2019). El uso del Google Classroom como complemento de la capacitación presencial a docentes de nivel primario. Revista Iberoamericana De Tecnología En Educación y Educación En Tecnología, (24), 79-90. https://doi.org/10.24215/18509959.24.e09

Labayen, M., Vea, R., Flórez, J., Guillén-Gámez, F. D., \& GarcíaMagariño, I. (2014). Smowl: a tool for continuous student validation based on face recognition for online learning [Comunicación]. Edulearn14 Proceedings. 6th International Conference on Education and New Learning Technologies, Barcelona, España. https://library.iated.org/view/LABAYEN2014SMO

López Álvarez, D. (2013). Si elimino el examen ¿mis alumnos dejarán de aprender? Una experiencia de diseño de actividades educativas alternativas al examen. ReVisión, 6(2),

$10-17$. http://hdl.handle.net/2117/20673

Moodle (2016). Gestionando un curso Moodle. Moodledocs. https://docs.moodle.org/all/es/Gestionando_un_curso_M oodle

Moodle (2021). Blackboard Collaborate. Plugins. https://moodle.org/plugins/mod collaborate

Moreno, F., \& Santiago, R. (2003). Formación online: guía para profesores universitarios. Logroño: Universidad de la Rioja.

https://www.researchgate.net/publication/39207293

Nadezhda, G. (2020). Zoom technology as an effective tool for distance learning in teaching English to medical students. Бюллетень науки и практики, 6(5), 457-460. https://doi.org/10.33619/2414-2948/54/61
Periódico La Rioja (2019). La UNIR comienza el curso con 45.000 estudiantes de 90 países. La Rioja. https://www.larioja.com/la-rioja/unir-comienza-curso20191118110930-nt.html

Piaget, J. (1975). Seis estudios de psicología. Barral Editores.

Pruneda, R. E. (2020). Moodle: gestión de contenidos online. Ediciones de la Universidad de Castilla-La Mancha.

Questroom School of Bussiness (2016). Respondus Lockdown Browser: Student quick start guide. http://questromworld.bu.edu/tech/files/2016/05/Respond us-LockDown-Browser_Student_Guide-5.6.2016.pdf

Romero, M. R., Castejón, F. J, López, V. M. y Fraile A. (2017). Evaluación formativa, competencias comunicativas y TIC en la formación del profesorado. Comunicar: Revista Científica Iberoamericana de Comunicación $y$ Educación, 25(52), 73-82. https://doi.org/10.3916/C522017-07

Ros Martínez de Lahidalga, I. (2008). Moodle, la plataforma para la enseñanza y organización escolar. http://hdl.handle.net/10810/6876

Trujillo, F. (2015). Aprendizaje basado en proyectos. Infantil, Primaria y Secundaria. Ministerio de Educación.

Universidad Católica de Ávila (2020a). La UCAV afronta los exámenes de junio con un seguro sistema de evaluación online. https://www.ucavila.es/la-ucav-afronta-losexamenes-de-junio-con-un-seguro-sistema-deevaluacion-online/

Universidad Católica de Ávila (2020b). Impreso de cesión de derechos de imagen. [Archivo de texto] https://www.ucavila.es/downloads/Instancias/DIPI.UCAV.docx

Universidad Católica de Ávila (2021). Grado en Maestro de Educación Primaria, modalidades de estudio. https://www.ucavila.es/grado-en-maestro-en-educacionprimaria/

Universidad Internacional de la Rioja. (2021). Misión y valores. https://www.unir.net/universidad-online/mision-valores/

Universidad Internacional de Valencia (2021a). Quiénes somos. https://www.universidadviu.com/es/quienes-somos

Universidad Internacional de Valencia (2021b). Normativa. https://www.universidadviu.com/es/normativa

Universidad Internacional de Valencia (2020). Plan adaptación COVID-19 para el curso 2019-2020. https://www.universidadviu.com/download/file/19154

Ximenes, A. C. (2020). Virtual Learning Environment: Report on the Moodle Management Experience in Public Higher Education School. In Learning Styles and Strategies for Management Students (pp. 232-243). IGI Global.

Zhang, M. (2016). Teaching with Google Classroom. Packt Publishing Ltd. 